Ann. Zootech., I966, 15 (3), 253-257.

\title{
L'INDICE DE FIBROSITE DES FOINS : MESURE ET RELATIONS AVEC LA VALEUR ALIMENTAIRE
}

\author{
M. CHENOST \\ Station de Recherches sur l'Élevage des Ruminants, \\ Centre de Recherches zootechniques et vétérinaires sur les Ruminants, \\ 6.3 - Theix, près Clermont-Ferrand
}

\section{SOMMAIRE}

Nous avons mis au point une méthode permettant d'apprécier le degré de fibrosité des foins par la mesure de l'énergie électrique nécessaire au broyage d'un échantillon de $5 \mathrm{~g}$ de foin. Cet indice de fibrosité présente une très grande amplitude de variation (de I à 8), ce qui permet de différencier des foins de caractéristiques assez voisines, malgré une reproductibilité encore médiocre.

L'indice de fibrosité des 25 foins étudiés présente des relations très étroites $(r>0,90)$ avec la digestibilité et avec la quantité de ces foins qu'ingèrent des moutons. Il permet ainsi d'estimer leur valeur alimentaire de façon plus satisfaisante que la cellulose brute. Cet indice de fibrosité peut constituer un test simple et rapide d'estimation de la valeur alimentaire des fourrages.

\section{INTRODUC'TION}

Au cours du premier cycle de croissance, la composition morphologique, histologique et chimique des plantes fourragères évolue de façon plus ou moins rapide ; il en est de même atı cours des cycles de croissance ultérieurs mais de façon moins nette, surtout pour les cycles feuillus des graminées. Corrélativement la valeur alimentaire de ces plantes diminue plus ou moins rapidement avec l'âge. On a depuis longtemps cherché à relier la digestibilité des plantes à leur composition chimique plus particulièrement à leur teneur en résidus membranaires (cellulose Weende, lignocellulose) ou à des constituants membranaires mieux définis (cellulose, lignine...).

L'aspect extérieur d'une plante se modifie également lorsqu'elle vieillit : l'importance et la dureté de ses tiges augmentent et elle devient plus grossière et plus fibreuse. 
Nous avons pensé qu'il devait être possible de mesurer ce " degré de fibrosité " et de le relier ensuite à la valeur alimentaire de la plante. Par analogie avec la mesure de la tendreté de la viande, (MrYADA et TAPPEL, I956), nous avons estimé ce degré de fibrosité par la mesure de la quantité d'énergie électrique nécessaire pour broyer une certaine quantité de fourrage sec dans des conditions bien définies. Il existe très peu d'études analogues; seuls à notre connaissance, Troeisen et BIGSBY (I964) au Canada, ont tout récemment cherché à relier l'acceptabilité des foins à l'aptitude de ces foins à se réduire en particules fines après une mastication artificielle.

\section{MATÉRIEL E'T MÉTHODE}

Parmi un ensemble de foins dont DEMARQuility mesurait la digestibilité sur des moutons pendant l'hiver I964-I965, nous avons choisi 25 foins de valeur alimentaire et d'aspect physique différents : le coefficient de digestibilité de la matière organique variait de 51,4 à 79,6 p. roo et la quantité de matière sèche ingérée de 37,0 à $87,9 \mathrm{~g} / \mathrm{kg} \mathrm{P} \mathrm{P}^{0,75}$. Nous appelons " indice de fibrosité " d'un foin l'énergie électrique nécessaire pour broyer $5 \mathrm{~g}$ de ce foin dans les conditions suivantes:

Un échantillon de 5 à Io $\mathrm{kg}$ de foin est haché dans un hache-paille (Koela type Sieger GO 3I5 K) puis grossièrement broyé dans un broyeur (Promill type B. $4 \mathrm{C}$ de $5 \mathrm{CV}$.) équipé d'une grille à mailles de $4 \mathrm{~mm}$ de diamètre. Le broyat obtenu est homogénéisé et une fraction de $300 \mathrm{~g}$ est mise à l'étuve à $70^{\circ}$ pendant 24 heures. Des prises de $5 . \mathrm{g}$ de ce produit sec sont introduites de façon brutale dans un broyeur de laboratoire Culatti équipé d'une grille à mailles de I mm fonctionnant en courant alternatif $220 \mathrm{~V}$ monophasé. Ce broyeur est branché en série avec un wattmètre enregistreur ( ${ }^{1}$ ) Richard qui permet d'enregistrer sur papier les variations de la puissance électrique consommée par le moteur du broyeur. Nous enregistrons ainsi 2 courbes pour chaque prise, l'une représente les variations de la puissanca consommée pour broyer cette prise de $5 \mathrm{~g}$ et l'autre, la puissance consommée lorsque le broyeur tourne à vide une fois le broyage terminé. L'énergie électrique dépensée (exprimée en $\mathbf{I} / \mathbf{I O}^{\mathrm{e}}$ de watt. heure) pour broyer la prise est proportionnelle à la surface comprise entre ces deux courbes. La forme des diagrammes obtenus dépend de la façon dont on introduit la prise dans le broyeur ; nous avons choisi d'introduire chaque prise d'une façon brutale, ce qui est plus facile à reproduire qu'une introduction lente.

\section{RÉSULTATS ET DISCUSSION}

La reproductibilité de la mesure est pour l'instant médiocre. Le coefficient de variation obtenu avec 6 répétitions par foin varie de 3 , I à 20,9 p. Ioo pour l'ensemble des 25 échantillons. Une analyse statistique portant sur 7 échantillons montre par ailleurs que les coefficients de variation obtenus avec $\mathbf{2} 2$ répétitions ne sont pas significativement inférieurs à ceux obtenus avec 6 répétitions.

(1) Nous remercions M. B.-L. Dumont qui a bien voulu nous prêter cet appareil pour effectuer nos mesures. 
Les limites de variation de 1'indice de fibrosité sont très grandes (de I,34 à Io,66) et bien supérieures aux limites de variation de la digestibilité ; la plus petite différence significative au seuil $\mathrm{P}<0,05$ est de $0,23 \frac{\mathrm{W} . \mathrm{h}}{\mathrm{IO}}$. On peut donc différencier de façon satisfaisante des foins de caractéristiques voisines et tolérer dans une certaine mesure une reproductiblité médiocre.

L'indice de fibrosité des foins présente des relations intéressantes d'une part avec leur digestibilité (fig. I), et d'autre part avec la quantité qu'en ingèrent des

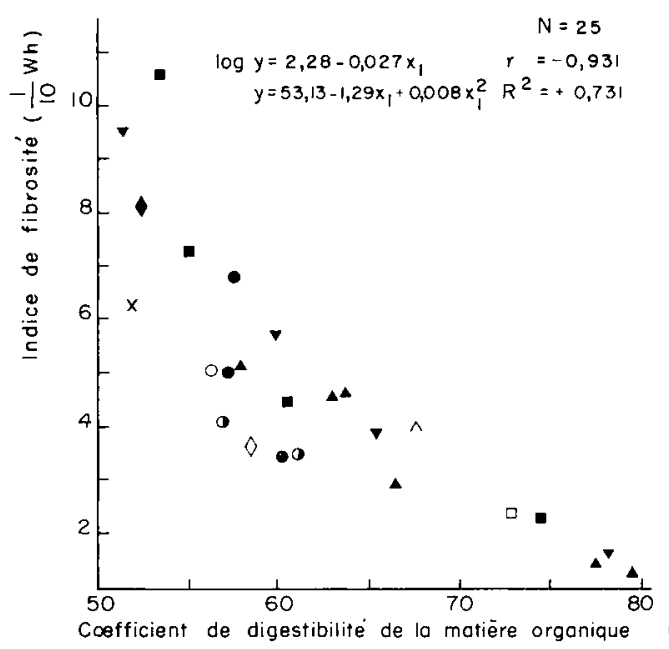

(p. 100)

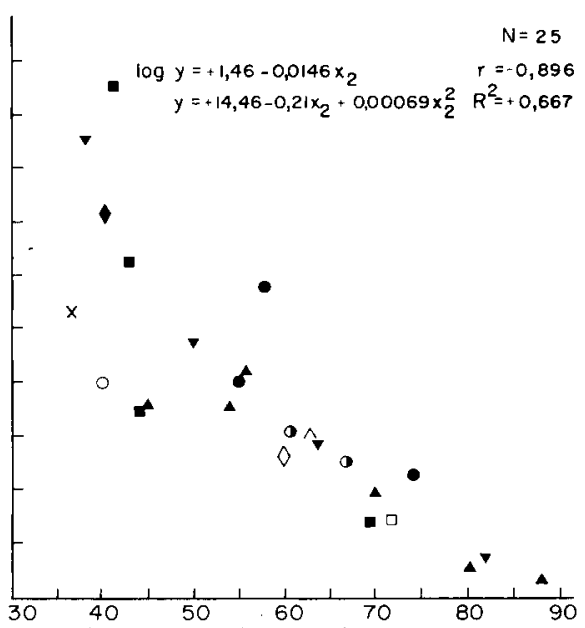

Quantité de matiēre sēche ingérée par des moutons $\left(\mathrm{g} / \mathrm{j} / \mathrm{kg} \mathrm{P}^{\mathrm{O}, 75}\right)$

FIG. I. - Relation entre l'indice de fibrosité $(Y)$ des FIG. 2. - Relation entre lindice de fibrosité (Y) foins et le coefficient de digestibilité de la malière organique $\left(X_{1}\right)$. et la quantité de matière sèche $\left(X_{2}\right)$ volontairement ingérée par des moutons.
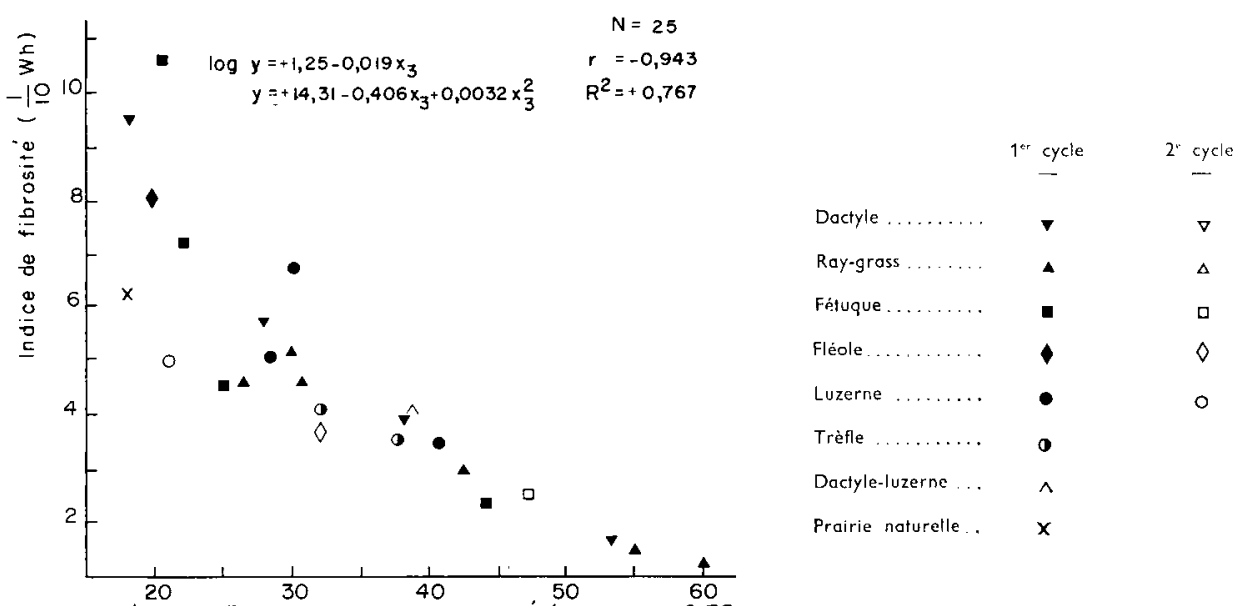

FIG. 3. - Relation entre l'indice de fibrosité $(Y)$ et la quantité de matière organique $\left(X_{3}\right)$ digestible ingérée par des moutons. 
moutons alimentés ad libitum (fig. 2). Par suite, cet indice de fibrosité est en liaison avec la quantité de matière organique digestible ingérée qui caractérise au mieux la valeur alimentaire (fig. 3). Les liaisons observées sont étroites et curvilinéaires Elles sont d'ailleurs plus étroites lorsqu'on ne considère que des foins de même nature, par exemple les graminées du i er cycle.

\section{TABLEAU I}

Comparaison de l'estimation de la digestibilité de la matière organique $\left(X_{1}\right)$, de la quantité de matière sèche ingérée $\left(X_{2}\right)$,

de la quantité de matière organique digestible ingérée $\left(X_{3}\right)$ à partir de l'indice de fibrosité $(Y)$

et à partir des critères généralement utilisés (cellulose brute $\left(X_{4}\right)$ ) et digestibilité de la matière organique

\begin{tabular}{|c|c|c|c|}
\hline Critères & Équation de régression & $\begin{array}{c}\text { Coefficient } \\
\text { de corrélation }\end{array}$ & $\begin{array}{c}\text { Écart-type } \\
\text { à partir } \\
\text { de la régression }\end{array}$ \\
\hline $\begin{array}{l}\text { Indice de fibrosité } \\
\text { Cellulose brute }\end{array}$ & $\begin{array}{l}\text { Digestibilité de la matière organique (p. 100) } \\
\begin{aligned} \mathrm{X}_{1}=82,19-32,4 \log \mathrm{Y} \\
\mathrm{X}_{1}=89,62-8,75 \mathrm{Y}+0,51 \mathrm{Y}^{2} \\
\mathrm{X}_{1}=76,18+0,377 \mathrm{X}_{4}\end{aligned}\end{array}$ & $\begin{aligned} r & =-0,931^{* *} \\
\mathrm{R}_{2} & =+0,877^{* *} \\
r & =-0,756^{* *}\end{aligned}$ & $\begin{array}{l}3,13 \\
3,07 \\
5,16\end{array}$ \\
\hline $\begin{array}{c}\text { Indice de fibrosité } \\
\text { Digestibilité } \\
\text { de la matière organique } \\
\text { Cellulose brute }\end{array}$ & $\begin{array}{l}\begin{array}{c}\text { Quantité de matière sèche ingérée } \\
(\mathrm{g} / \mathrm{jour} / \mathrm{kg} \mathrm{P} 0,75)\end{array} \\
\mathrm{X}_{2}=91,74-55,0 \log \mathrm{Y} \\
\mathrm{X}_{2}=102,59-13,92 \mathrm{Y}+0,77 \mathrm{Y}^{2} \\
\mathrm{X}_{2}=-36,16+1,511 \mathrm{X}_{1} \\
\mathrm{X}_{2}=98,31-1,098 \mathrm{X}_{4}\end{array}$ & $\begin{aligned} r & =-0,896^{* *} \\
\mathbf{R}_{2} & =+0,827^{* *} \\
r & =+0,859^{* *} \\
r & =-0,616^{* *}\end{aligned}$ & $\begin{array}{r}6,55 \\
6,41 \\
7,77 \\
11,80\end{array}$ \\
\hline $\begin{array}{c}\text { Indice de fibrosité } \\
\text { Digestibilité } \\
\text { de la matière organique } \\
\text { Cellulose brute }\end{array}$ & 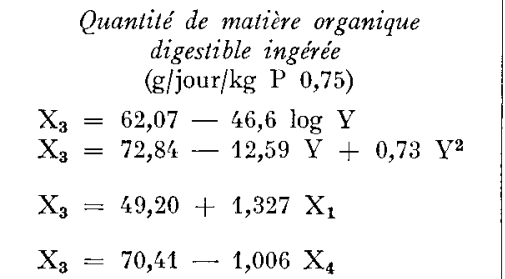 & $\begin{aligned} r & =-0,943^{* *} \\
\mathrm{R}_{2} & =+0,912^{* *} \\
r & =+0,935^{* *} \\
r & =-0,699^{* *}\end{aligned}$ & $\begin{array}{l}4,05 \\
3,70\end{array}$ \\
\hline
\end{tabular}

$\mathrm{P}<0,01$

Nous pouvons rendre ces relations linéaires en prenant le logarithme de l'indice de fibrosité. Cependant, cette transformation logarithmique modifie la distribution des données qui ne sont plus distribuées normalement. On peut penser que cela est peut-être dî̀ au nombre insuffisant de données, en particulier dans la zone des digestibilités comprises entre $65 \mathrm{p}$. Ioo et $80 \mathrm{p}$. Ioo. Nous donnons donc les équations liant l'indice de fibrosité aux différents critères de la valeur alimentaire des foins soit sous forme curvilinéaire, soit sous forme logarithmique (fig. I, 2 et 3). 
La digestibilité des 25 foins étudiés peut être estimée à partir de 1'indice de fibrosité d'une façon plus précise qu'à partir de la teneur en cellulose brute (tabl. I) qui est pourtant le critère le plus utilisé encore à l'heure actuelle. Cette relation n'est pas étonnante : la digestibilité d'un fourrage dépend en effet étroitement de l'importance et de la lignification des membranes qui sont deux caractéristiques responsables de sa "fibrosité".

l)e même, la quantité de fourrage volontairement ingérée par les moutons peut être estimée à partir de l'indice de fibrosité d'une façon plus précise qu'à partir de la digestibilité (tabl. I). Elle dépend en effet de la vitesse de dégradation de la masse qui encombre le rumen en particules suffisamment fines pour que celles-ci puissent transiter vers les compartiments ultérieurs. Il n'est donc pas étonnant qu'il existe un parallèle entre l'énergie nécessaire au broyage des fourrages et leur vitesse de dégradation en particules fines, donc la quantité qui en est ingérée. En définitive, l'indice de fibrosité permet d'estimer la valeur alimentaire des fourrages caractérisée par la quantité de matière organique digestible ingérée, avec une précision qui est supérieure à celle obtenue avec la teneur en cellulose brute.

La mesure de l'indice de fibrosité semble satisfaisante et peut constituer un test simple et rapide d'estimation de la valeur alimentaire des fourrages secs ; cependant il est nécessaire d'améliorer sa reproductibilité et d'augmenter la précision des relations observées. Pour cela, nous utiliserons, lors de nos prochaines mesures, un appareil qui permettra de mesurer non plus l'énergie électrique mais l'énergie mécanique réellement consommée par le broyage des fourrages. Nous éviterons ainsi l'erreur liée à l'introduction brutale de la prise de foin qui entraine une variation de la vitesse. de rotation du moteur électrique et par conséquent une variation de son rendement.

Reçu pour publication en juin 1966.

\section{SUMMARY}

INDEX OF FIBROUSNESS OF HAYS ; ITS MEASUREMENT

AND RELATION TO FEEUING VALUE

A method is described by which the degree of fibrousness of hay can be shown by measurement of the electrical energy needed to grind a sample of $5 \mathrm{~g}$ hay. This index varies widely from $\mathrm{I}$ to 8 so that hays of quite similar characteristics may be distinguished from each other.

The index of fibrousness of 25 hays studied was very closely related $(r>70.90)$ to digestibility and to intake of the hays by sheep. It was thus possible to estimate their feeding value more satisfactorily than from crude fibre. The index of fibrousness could be a simple and rapid method for estimation of the feeding value of forages.

\section{RÉFÉRENCES BIBI,IOGRAPHIQUES}

Miyada D. S., Tappel A. L., i 956 . Meat tenderization. I. Two mechanical devices for measuring texturer. Food Technol., 10, $14^{2-1} 45$.

Troelsen J. E., Bigsby F. W., 1964. Artificial mastication. A new approach for predicting voluntray forage consumption by ruminants. J. anim. Sci., 23, II39-1 I42. 